Bio - grafía. Escritos sobre la Biología y su Enseñanza. ISSN 2027-1034

Edición Extraordinaria. p.p. 351 - 362

Memorias del IX Encuentro Nacional de Experiencias en Enseñanza de la Biología y la Educación Ambiental. IV Congreso Nacional de Investigación en Enseñanza de la Biología.

\title{
EL ÉNFASIS EN BIOTECNOLOGÍA COMO ESCENARIO DE INCLUSIÓN Y DESARROLLO DE UNA ACTITUD EMPRENDEDORA
}

\author{
Morales Bermúdez Javier Mauricio'
}

\section{RESUMEN}

En la actualidad, una amplia proporción de jóvenes egresados de la educación media deben enfrentarse a la búsqueda de empleo y a la inminente necesidad de generar ingresos para su subsistencia y la de sus familias, sin haber recibido una formación orientada para ello. Ante esta situación, el énfasis en Biotecnología de la educación media en la jornada nocturna del colegio La Amistad IED surge como una oportunidad y apuesta de fomentar la inclusión del joven y adulto desde su proyecto de vida y sus necesidades e intereses a partir de una formación flexible, participativa y potenciadora de actitudes emprendedoras y capacidades ciudadanas a través de la articulación del conocimiento biotecnológico escolar con la vida experiencial de los estudiantes. En este orden de ideas, el campo de la Biotecnología, que como asignatura se trabaja en los ciclos 5 y 6 (Grados 10 y 11) busca acercar a los estudiantes el conocimiento científico de manera contextualizada con el propósito de proponer en marcha proyectos Biotecnológicos con sentido emprendedor. Los avances en el aprendizaje de la Biotecnología y en el desarrollo de actitudes emprendedoras se han hecho visibles en la participación de los estudiantes en escenarios de emprendimiento y en eventos de divulgación científica y tecnológica. Así las cosas, a través del trabajo por proyectos desde un aprendizaje situado, se ha alcanzado la reflexión sobre asuntos sociocientíficos, propiciando al mismo tiempo la conexión entre el aprendizaje de las ciencias y los problemas reales del mundo con apuestas prácticas en la solución de problemas.

PALABRAS CLAVE: Biotecnología, inclusión, emprendimiento, ambientes de aprendizaje, educación de adultos.

\footnotetext{
1 Magister en Educación con énfasis en Investigación, Universidad de Sherbrooke, QuebecCanadá, Magister en Dirección Universitaria, Universidad de los Andes, Especialista en Multimedia Educativa. Directivo Universitario, Tutor Científico de la Asociación Colombiana para el Avance de la Ciencia, ACAC, Docente de Biotecnología, Química y Biología del Colegio Distrital La Amistad, Área de Ciencias Naturales, Jornada Nocturna. Ciencialudica2009@gmail.com
} 
Bio - grafía. Escritos sobre la Biología y su Enseñanza. ISSN 2027-1034

Edición Extraordinaria. p.p. 351 - 362

Memorias del IX Encuentro Nacional de Experiencias en Enseñanza de la Biología y la

Educación Ambiental. IV Congreso Nacional de Investigación en Enseñanza de la Biología.

\begin{abstract}
At present, a large proportion of young graduates of secondary education must face the search for employment and the imminent need to generate income for their subsistence and that of their families, without having received training aimed at it. Faced with this situation, the emphasis in Biotechnology arises as an vopportunity and commitment for the middle school of the night of the school La Amistad IED, to promote the inclusion of the young and adult from their project of life and their needs and interests from a flexible, participatory and empowering of entrepreneurial attitudes and citizen capacities through diverse learning environments that articulate the biotechnological knowledge school with the students' experiential life. In this context, the field of Biotechnology, which is a subject of work in cycles 5 and 6 (Grades tenth and eleventh) seeks to bring students scientific knowledge in a contextualized way with the purpose of proposing biotechnological projects in progress with entrepreneurial sense. In this way, the advances in the learning of the sciences and the development of entrepreneurial attitudes have become visible in the participation of the students in entrepreneurial scenarios and in events of scientific and technological diffusion. Thus, through the work by projects from a situated learning, has reached the reflection on socio-scientific issues, while at the same time facilitating the connection between the learning of the sciences and the real problems of the world with practical bets in the solution of problems.
\end{abstract}

KEY WORDS: Biotechnology, inclusion, entrepreneurship, learning environments, adult education.

\title{
INTRODUCCIÓN
}

La Unesco (2005) precisa el concepto de inclusión como un enfoque que responde positivamente a la diversidad de las personas y a las diferencias individuales, entendiendo que la diversidad no es un problema, sino una oportunidad para el enriquecimiento de la sociedad, a través de la activa participación en la vida familiar, en la educación, en el trabajo y en general en todos los procesos sociales, culturales y en las comunidades.

En este sentido, se pone de manifiesto la oportunidad de generar en los estudiantes de la jornada nocturna del Colegio La Amistad IED, ubicado en la localidad de Kennedy (Bogotá - Colombia) una actitud emprendedora a través del trabajo realizado en el énfasis en Biotecnología, que como énfasis de la educación Media $^{2}$, se desarrolla en los ciclos 5 y 6 (Grados 10 y 11) a través de proyectos biotecnológicos con sentido emprendedor. El desarrollo de las actividades propias del énfasis en Biotecnología ha posibilitado tanto la

\footnotetext{
${ }^{2}$ Los tres niveles de educación formal en Colombia son: preescolar, educación básica primaria y básica secundaria, y educación media la cual contempla los dos últimos grados de la educación secundaria. La educación media académica permitirá al estudiante, según sus intereses y capacidades, profundizar en un campo específico de las ciencias, las artes o las humanidades y acceder a la educación superior (Ley 115 Art. 29).
} 


\section{Bio - grafía. Escritos sobre la Biología y su Enseñanza. ISSN 2027-1034}

Edición Extraordinaria. p.p. 351 - 362

Memorias del IX Encuentro Nacional de Experiencias en Enseñanza de la Biología y la Educación Ambiental. IV Congreso Nacional de Investigación en Enseñanza de la Biología.

construcción de conocimientos científicos implicados en aplicaciones biotecnológicas, como el ejercicio de los derechos humanos desde la participación en proyectos de emprendimiento, ambientales, de servicio social y eventos académicos que han permitido la manifestación de las capacidades ciudadanas, el respeto a la diferencia y la construcción colectiva de escenarios de aprendizaje y liderazgo. Iniciativas que son señales de una educación en y para la inclusión sin fronteras y con diálogos diversos en la construcción de nuevos caminos.

Cabe destacar que los estudiantes de la jornada ${ }^{3}$ nocturna son en su gran mayoría personas con un gran saber popular derivado de sus diversas experiencias de vida; de estratos socioeconómicos 1, 2 y 3 principalmente; entre 15 y 65 años de edad y que suelen presentar una variedad de problemáticas y conflictos familiares y sociales de gran consideración.

De manera tal que la pregunta que se busca responder en la experiencia que presentamos a continuación es: ¿cómo crear a partir de las actividades del énfasis en Biotecnología un espacio para todos en donde, a partir del respeto y potenciación de la diferencia, cada cual tenga las mismas posibilidades de generar proyectos de emprendimiento?

La apuesta desde el énfasis se centra entonces en fomentar la inclusión del joven y adulto desde su proyecto de vida y sus necesidades e intereses a partir de una formación flexible, participativa y potenciadora de capacidades ciudadanas a través de diversos ambientes de aprendizaje que articulan un conocimiento biotecnológico escolar con la vida experiencial de los estudiantes. De lo que se trata es de dar la mirada hacia la Inclusión en la cual como lo plantea Orjuela (2013) "Se pasa del sujeto y sus limitaciones a la concentración en el ambiente y los apoyos que éste provea, ahora el interés no es lo individual sino la especie, el grupo, es parte del ejercicio de lo biopolítico".

La incorporación de la biotecnología en la educación básica y media en Colombia y el mundo

Para precisar el término Biotecnología, cabe mencionar la definición más aceptada internacionalmente, según la cual la Biotecnología es "toda aplicación tecnológica que utilice sistemas biológicos y organismos vivos o sus derivados para la creación o modificación de productos o procesos para usos específicos" (Convention on Biological Diversity, Article 2. Use of Terms, United Nations. 1992).

Como bien lo expone Frid, 2009: "La Biotecnología es una disciplina que se está integrando a la enseñanza escolar actual. Por ser un tema novedoso en la didáctica de las ciencias, plantea interrogantes acerca de los conceptos teóricos y prácticos que debería abarcar, su incorporación a la planificación, la formación del docente, y la disponibilidad y el acceso a recursos educativos específicos".

Hoy en día se puede apreciar cómo se hacen visibles estamentos internacionales y países que lideran programas de enseñanza de la Biotecnología en los diferentes niveles

${ }^{3}$ El Colegio presta su servicio educativo a los estudiantes en tres jornadas: mañana, tarde y noche. 


\title{
Bio - grafía. Escritos sobre la Biología y su Enseñanza. ISSN 2027-1034
}

\section{Edición Extraordinaria. p.p. 351 - 362}

\author{
Memorias del IX Encuentro Nacional de Experiencias en Enseñanza de la Biología y la \\ Educación Ambiental. IV Congreso Nacional de Investigación en Enseñanza de la \\ Biología.
}

educativos, entre los cuales cabe mencionar la E.I.B.E. (Iniciativa Europea para la Enseñanza de la Biotecnología), que está conformada por varios países (Bulgaria, Polonia, República Checa, Grecia, Suiza, Holanda), organizaciones internacionales de países como Dinamarca, España, Estados Unidos e Irlanda y los programas de educación en biotecnología para el público en general y para los profesores de ciencias propuestos por el conjunto de la Comunidad Europea (Padilla, et al., 1997).

En el continente Latinoamericano también se pueden encontrar diversas estrategias metodológicas y didácticas para la enseñanza de la biotecnología tanto en educación secundaria como al nivel universitario, tanto en países pertenecientes a la Red BioEDUCAR (Argentina, Brasil, Bolivia, Chile, Cuba, Ecuador, España, México y Venezuela), como en países que están por fuera de ella, lo cual pone en evidencia la importancia que poco a poco ha cobrado la Biotecnología para el sector educativo (Espinel, 2015).

Para el caso de Colombia, la investigación y las aplicaciones biotecnológicas y la formación académica, aún son escasas; el Instituto de Biotecnología de la Universidad Nacional de Colombia (IBUN) está realizando esfuerzos por socializar los conocimientos sobre las herramientas de uso frecuente en la Biotecnología con el fin de llevar a cabo programas integrales para actualizar los conocimientos de los profesores del área de ciencias naturales (Parra y Reguero, 2001). Por su parte, la Línea de Investigación en Biotecnología y Educación de la Universidad Pedagógica Nacional propone la Biotecnología como elemento importante para la enseñanza de las ciencias; han descrito e interpretado proyectos escolares de corte biotecnológico en varias instituciones de educación básica y media, y han propuesto unidades didácticas bajo el modelo de enseñanza-aprendizaje como investigación (Espinel, 2015), queriendo de esta manera acercar al estudiante a la naturaleza de las ciencias y el establecimiento de las relaciones ciencia-tecnología-sociedad.

Este enfoque ciencia-tecnología-sociedad (CTS) para la enseñanza de las ciencias Naturales es al mismo tiempo un campo de estudio e investigación y, sobre todo, una propuesta educativa innovadora de carácter general (Acevedo, 1997; Vázquez, 1999 citados por Gordillo, 2009). Desde esta perspectiva de enseñanza se busca una mejor comprensión de la ciencia y la tecnología en su contexto social por lo que se abordan las relaciones mutuas entre los desarrollos científicos y tecnológicos y los procesos sociales. Como propuesta educativa general el enfoque CTS constituye un nuevo planteamiento radical del curriculum en todos los niveles de enseñanza, con la principal finalidad de generar una formación en conocimientos y, especialmente, en valores que favorezca la participación ciudadana responsable y democrática en la evaluación y el control de las implicaciones sociales de la ciencia y la tecnología.

\section{Políticas Educativas en Colombia que incluyen a la Educación de Jóvenes y Adultos}

La Ley General de Educación Colombiana (Ley 115), en el Título III de Modalidades de Atención educativa a poblaciones, desarrolla cinco apartados dirigidos a la educación de personas con limitaciones y capacidades excepcionales; adultos; grupos étnicos; campesinos y rehabilitación social. 


\section{Bio - grafía. Escritos sobre la Biología y su Enseñanza. ISSN 2027-1034}

Edición Extraordinaria. p.p. 351 - 362

Memorias del IX Encuentro Nacional de Experiencias en Enseñanza de la Biología y la

Educación Ambiental. IV Congreso Nacional de Investigación en Enseñanza de la Biología.

En los últimos 13 años en Colombia se ha venido planteando el tema de la inclusión desde las políticas de gobierno. Así, con la revolución educativa se desarrolla el Programa de Inclusión con Calidad "Construyendo la capacidad Institucional para la Atención de la Diversidad", y se elabora un instrumento para medir el índice de inclusión en las instituciones educativas. Adicionalmente, se incorpora la atención por grupos poblacionales entre los cuales se encuentran los jóvenes y adultos iletrados.

El tema de inclusión ha sido parte también de las últimas políticas distritales, de forma tal que con el Plan de Desarrollo Distrital 2012-2016 "Bogotá Humana" se propone la reducción de la desigualdad y la discriminación mientras que, la Secretaria de Educación del Distrito (SED) plantea desde la Dirección de Inclusión e Integración de poblaciones, el programa de Enfoques Diferenciales en el cual incorpora la educación de adultos.

\section{METODOLOGÍA}

La experiencia educativa que presentamos constó de dos fases: Una fase de caracterización de la enseñanza de las ciencias en la Jornada Nocturna y selección del énfasis de Biotecnología para la educación media y, una fase de implementación del énfasis en Biotecnología, las cuales se describen a continuación:

- Caracterización de la enseñanza de las ciencias en la Jornada Nocturna del colegio la Amistad IED y selección del énfasis de Biotecnología para la educación media: Durante el año 2009 la comunidad de la jornada nocturna del colegio, decidió revisar, a través de una amplia discusión, la enseñanza de las ciencias en la institución. La revisión señalada permitió encontrar que la educación en ciencias carecía de contexto, no era pertinente, era poco flexible, tenía acceso reducido a los recursos institucionales y escasa participación en la toma de decisiones a nivel institucional. Se detectó además que la planeación de las clases se limitaba a desarrollar una lista de temas por asignatura, producto de un filtro de los planes de estudios desarrollados para los niños y jóvenes de las jornadas diurnas. Problemáticas que responden a un modelo educativo homogeneizador que no tiene en cuenta las características del adulto y su contexto, y que según Orjuela (2013), se corresponden con un modelo anatomopolitico, concentrado en el sujeto y la homogenización, en el que las preguntas se hacen desde el sujeto, su patología, su etiología, sus limitaciones y las maneras como él podría integrarse o llevar una vida lo más cercana a lo normalizado por la sociedad. La caracterización realizada condujó a plantear un nuevo enfoque en la enseñanza y aprendizaje de las ciencias en la jornada nocturna del colegio, con lo cual, se decidió implementar el proyecto de énfasis en Biotecnología presentado por el Licenciado Javier Morales Bermúdez como una propuesta curricular incluyente para la educación de jóvenes y adultos. La propuesta tuvo como antecedentes la revisión de los contenidos curriculares a partir del diagnóstico realizado en el año 2006; los lineamientos curriculares del MEN y las tendencias de la enseñanza de las Ciencias en la escuela. Así mismo, se tuvieron en cuenta la trayectoria institucional en el campo de la Biotecnología y, los laboratorios y la dotación de instrumentos y equipos 
Bio - grafía. Escritos sobre la Biología y su Enseñanza. ISSN 2027-1034

Edición Extraordinaria. p.p. 351 - 362

Memorias del IX Encuentro Nacional de Experiencias en Enseñanza de la Biología y la Educación Ambiental. IV Congreso Nacional de Investigación en Enseñanza de la Biología.

obtenidos a través de las experiencias de profesores de ciencias naturales de las jornadas mañana y tarde.

La apuesta desde la Biotecnología busca fomentar la inclusión del joven y adulto desde su proyecto de vida y sus necesidades e intereses, a partir de una formación flexible, participativa y potenciadora de capacidades ciudadanas a través de diversos ambientes de aprendizaje que articulen un conocimiento biotecnológico escolar con la vida experiencial de los estudiantes y por ende educativa. En este sentido, la pregunta orientadora que surge es: ¿cómo crear a partir de las actividades del énfasis en Biotecnología un espacio para todos en donde, a partir del respeto y potenciación de la diferencia, cada cual tenga las mismas posibilidades de generar proyectos de emprendimiento?

- Implementación del énfasis en Biotecnología: En el año 2010 se dio inicio a la implementación del énfasis en Biotecnología, encaminado a generar en los estudiantes jóvenes y adultos una actitud investigativa y emprendedora a través del trabajo en el campo de la Biotecnología, que como asignatura se trabaja en los ciclos 5 y 6 (Grados 10 y 11). Lo anterior ha conducido a indagar sobre pedagogías incluyentes para acercar el conocimiento científico a los estudiantes de manera contextualizada y, finalmente a la puesta en marcha de proyectos Biotecnológicos con sentido emprendedor.

Actualmente el énfasis en Biotecnología se desarrolla en cuatro módulos así, Módulo 1: Fundamentación en Biotecnología, Módulo II: Bioprocesos, Módulo III: Cultivo In Vitro de Tejidos Vegetales y Módulo IV: Proyecto Integrador. Módulos que responden a su vez a tres ejes transversales, Eje 1: Investigación, Eje 2: Calidad y Ambiente y Eje 3: Emprendimiento; con los cuales se busca darle sentido y alcance a la propuesta y al fortalecimiento del concepto de énfasis en Biotecnología como un espacio de creación, innovación e inclusión.

El enfoque metodológico que orienta la implementación del énfasis se fundamenta en los lineamientos de la metodología Investigación-Acción-Participación, (IAP), la cual, de acuerdo con Kemmis y McTaggart, (1988, p.283) "surge de la clarificación de preocupaciones compartidas en un grupo, intentando descubrir que puede hacerse, para llegar a adoptar un proyecto de grupo", con lo cual, adicionalmente se asume el enfoque basado en el aprendizaje situado, es decir, un aprendizaje producto de la interacción entre agentes y elementos del entorno; donde la utilización y adecuación de las herramientas con el medio circundante se tornan como premisas particulares en la construcción de conocimiento (Paz, 2007).

En este orden de ideas, la implementación del énfasis en Biotecnología pretende el establecimiento de relaciones entre los aprendizajes sobre Biotecnología con la realidad inmediata de los estudiantes. Es decir, proporcionar un conocimiento contextualizado que le permita al estudiante ubicarse en una realidad y apropiarse de unos conocimientos con un propósito definido en las dinámicas de transformación de 
Bio - grafía. Escritos sobre la Biología y su Enseñanza. ISSN 2027-1034

Edición Extraordinaria. p.p. 351 - 362

Memorias del IX Encuentro Nacional de Experiencias en Enseñanza de la Biología y la Educación Ambiental. IV Congreso Nacional de Investigación en Enseñanza de la Biología.

realidades. En este sentido, el desarrollo de actitudes investigativas y emprendedoras contribuyen a despertar el sentido crítico y propositivo de los estudiantes a partir de su entorno inmediato.

Desde el aprendizaje situado se aborda el trabajo en Biotecnología a partir de las siguientes premisas:

- El saber popular de los estudiantes: Esto es lo que piensan, sienten, sueñan y han vivido los estudiantes en su cotidianidad con relación en temas desarrollados en clase.

- El tratamiento de problemas socioculturales a través de los contenidos de carácter científico que fomente la participación de los estudiantes en comunidades de práctica.

- La capacidad de exploración y potenciación del espíritu emprendedor para aplicar los conocimientos en la generación de ideas emprendedoras.

- La implicación del estudiante con el pensamiento compartido con otras personas mediante el trabajo colaborativo.

Para ello, se ha desarrollado una ruta metodológica que establece un trabajo desde la lectura del contexto y sus problemáticas, abordadas desde el conocimiento en el campo de la Biotecnología, la formulación de preguntas que permitan develar un tipo de solución a tales problemáticas, el trabajo colaborativo en comunidades de aprendizaje, la aplicación de técnicas e instrumentos para generar productos y/o resultados y finalmente la socialización y aplicación de ideas de negocio en el sector real.

Las fases de la ruta metodológica implementada con los estudiantes jóvenes y adultos de la jornada nocturna se presentan a continuación:

Tabla 1. Fases de la ruta metodológica del énfasis en biotecnología

\begin{tabular}{|c|c|c|}
\hline Fase & Denominación & Descripción \\
\hline I & Sensibilización & $\begin{array}{l}\text { Estudios de necesidades del contexto con atención posible } \\
\text { desde la biotecnología }\end{array}$ \\
\hline II & Capacitación & $\begin{array}{l}\text { Abordaje conceptual y técnico de las áreas de aplicación de } \\
\text { la biotecnología }\end{array}$ \\
\hline III & $\begin{array}{l}\text { Planteamiento } \\
\text { Problemas }\end{array}$ & $\begin{array}{l}\text { Definición de la problemática que se va a atender de } \\
\text { manera colaborativa }\end{array}$ \\
\hline IV & $\begin{array}{l}\text { Alistamiento espacio de } \\
\text { trabajo }\end{array}$ & Adecuación espacio de trabajo e intervención \\
\hline
\end{tabular}


Bio - grafía. Escritos sobre la Biología y su Enseñanza. ISSN 2027-1034

Edición Extraordinaria. p.p. 351 - 362

Memorias del IX Encuentro Nacional de Experiencias en Enseñanza de la Biología y la

Educación Ambiental. IV Congreso Nacional de Investigación en Enseñanza de la Biología.

\begin{tabular}{|c|l|l|l|}
\hline V & $\begin{array}{l}\text { Implementación } \\
\text { procesos y Técnicas }\end{array}$ & $\begin{array}{l}\text { requerimientos de materiales. } \\
\text { Aplicación de métodos y técnicas para el montaje del } \\
\text { proyecto. }\end{array}$ \\
\hline VI & $\begin{array}{l}\text { Seguimiento } \\
\text { Evaluación }\end{array}$ y & $\begin{array}{l}\text { Protocolos de seguimiento y evaluación de procesos y } \\
\text { productos. }\end{array}$ \\
\hline VII & $\begin{array}{l}\text { Producción } \\
\text { Biotecnológica }\end{array}$ & $\begin{array}{l}\text { De acuerdo con la línea de trabajo se obtendrán productos } \\
\text { de interés biotecnológico (bioabonos, bioprocesos, Cultivos } \\
\text { In vitro, etc.) }\end{array}$ \\
\hline VIII & $\begin{array}{l}\text { Generación de Ideas } \\
\text { emprendedoras }\end{array}$ & $\begin{array}{l}\text { Aplicación del método Canvas }{ }^{4} \text { para la generación de ideas } \\
\text { de negocio y participación en la muestra de } \\
\text { emprendimiento. }\end{array}$
\end{tabular}

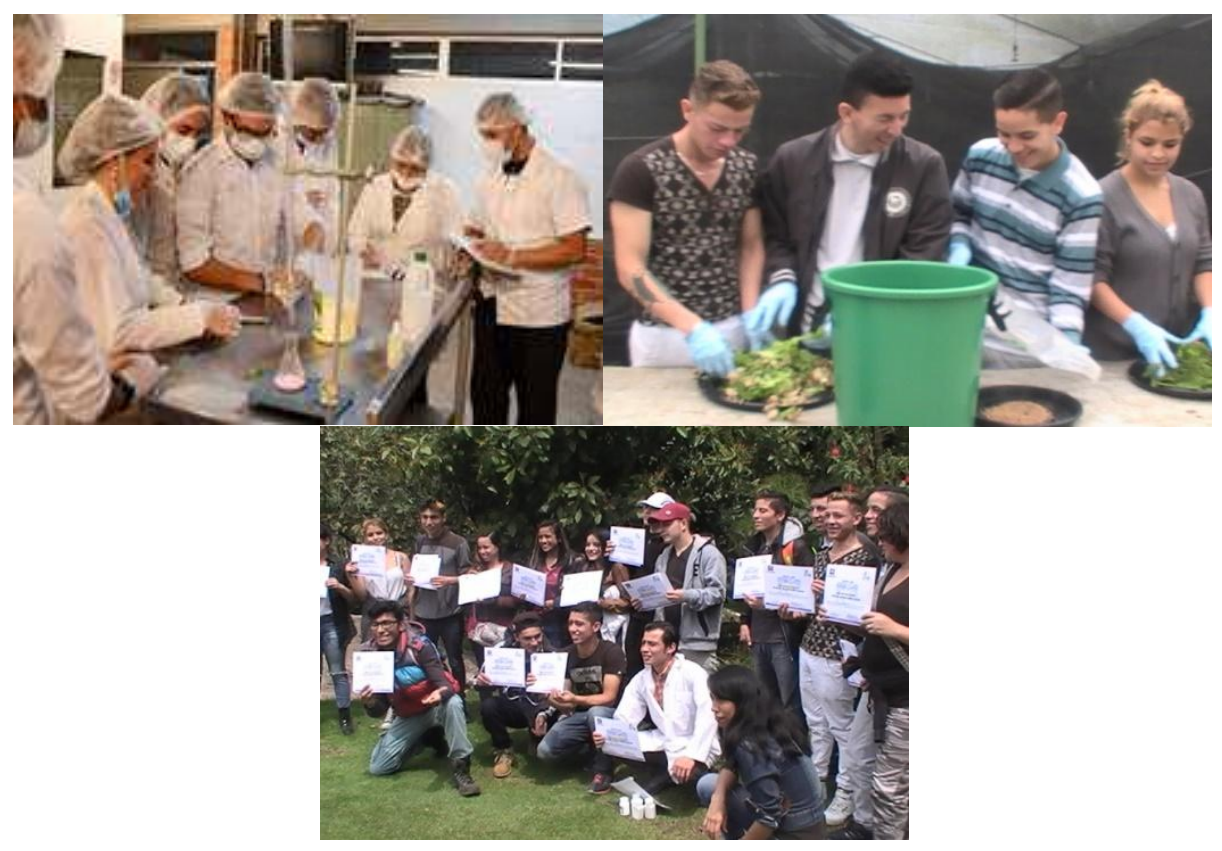

Fotos: Autor. Prácticas de Laboratorio y Salidas de campo.

En relación con las estrategias y actividades que llevaron a generar actitudes emprendedoras en los estudiantes se tienen: a. Talleres de emprendimiento, b. Exposición de las ideas de negocio en la Muestra Empresarial a nivel institucional. c. Asesorías en la consecución de recursos y presupuesto para consolidar y desarrollar el

\footnotetext{
${ }^{4}$ El método Canvas es una herramienta para generar modelos de negocios, que describe de manera lógica la forma en que las organizaciones crean, entregan y capturan valor.
} 


\title{
Bio - grafía. Escritos sobre la Biología y su Enseñanza. ISSN 2027-1034
}

\section{Edición Extraordinaria. p.p. 351 - 362}

\author{
Memorias del IX Encuentro Nacional de Experiencias en Enseñanza de la Biología y la \\ Educación Ambiental. IV Congreso Nacional de Investigación en Enseñanza de la \\ Biología.
}

Plan de Empresa. Es importante mencionar que para llevar a cabo esta propuesta se han desarrollado alianzas estratégicas con instituciones que han posibilitado la realización de visitas, prácticas y asesorías entre las cuales se encuentran el Servicio Nacional de Aprendizaje (Sena) del municipio de Mosquera, el Instituto de Biotecnología de la Universidad Nacional (IBUN), Corporación Colombiana de Investigación Agrícola (Corpoica), Levapán, las Universidades Católica de Colombia, INCCA de Colombia y de la Salle, la Cámara de Comercio de Kennedy y la Asociación Colombiana para el avance de la Ciencia.

Los trabajos y proyectos realizados por los estudiantes, han sido registrados en una bitácora y luego, a través de un informe se destacan los aciertos y aspectos a mejorar como insumos para la planeación del año siguiente. Cabe señalar además que cada año se realiza una evaluación integral de la propuesta y se ajustan contenidos y estrategias a partir de las sugerencias de los estudiantes y los resultados obtenidos.

\section{RESULTADOS Y DISCUSIÓN}

Con la intervención en la educación de jóvenes y adultos de los ciclos 5 y 6 de la jornada nocturna del colegio La Amistad IED, realizada a través del énfasis en Biotecnología se han podido alcanzar avances en los procesos de aprendizaje de las ciencias y en el desarrollo de actitudes emprendedoras así como de capacidades ciudadanas, evidentes y visibles en la participación de los estudiantes en escenarios como la feria de la ciencia anual, Expociencia y Expotecnología (2009, 2011, 2013 y 2015), encuentros con la ciencia organizados por la ACAC, Ferias de emprendimiento y otros eventos de divulgación científica y tecnológica.

Entre los proyectos de emprendimiento construidos se cuenta con 30 ideas de negocio en los últimos 6 años orientados en temáticas como alimentos funcionales, bioabonos, extracción de aceites vegetales, biocombustibles y biofertilizantes, cultivos in vitro de Orquídeas entre otros.

En cada uno de los proyectos desarrollados se partió de la sensibilización a la comunidad estudiantil desde su proyecto de vida, las problemáticas del contexto y las posibilidades que brinda el conocimiento biotecnológico para generar soluciones a partir de bioprocesos, a través de las cuales generar oportunidades de emprendimiento social y económico.

Un ejemplo de ello, es el proyecto de bioabonos en el que participaron grupos colaborativos que investigaron las problemáticas generadas por el inadecuado manejo de los residuos orgánicos en la localidad de Kennedy y la posibilidad de aplicar el conocimiento biotecnológico para aprovechar los residuos orgánicos transformándolos en bioabonos empleando procesos de compostaje y Técnicas como Bokashi EM. Desde la formación en emprendimiento, los estudiantes tuvieron el acompañamiento de la facultad de ingeniería industrial de la Universidad Católica de Colombia para elaborar técnicamente los procesos industriales para la producción de bioproductos y para acompañar en el desarrollo de la idea de negocio a partir del método Canvas. 


\title{
Bio - grafía. Escritos sobre la Biología y su Enseñanza. ISSN 2027-1034
}

\section{Edición Extraordinaria. p.p. 351 - 362}

\author{
Memorias del IX Encuentro Nacional de Experiencias en Enseñanza de la Biología y la \\ Educación Ambiental. IV Congreso Nacional de Investigación en Enseñanza de la \\ Biología.
}

Posteriormente, los estudiantes a través del trabajo en equipo, generan ideas de negocio que una vez avaladas son presentadas en la Muestra de Emprendimiento Institucional y en eventos académicos externos.

Los estudiantes también han logrado participar en discusiones relacionadas con temas de carácter científico y en la elaboración de medios de divulgación como el boletín de divulgación científica "Ecociencia", blogs, grupos de Facebook y el curso virtual de Biotecnología, lo que adicionalmente ha aumentado su acceso a las TIC en sus procesos formativos. Así mismo, han podido establecer una relación entre los aprendizajes adquiridos en el aula con la confrontación de los mismos con el sector real y la formulación de proyectos contextualizados, en un escenario de inclusión y respeto por la diversidad.

Entre los desempeños que se han podido detectar en los estudiantes se encuentran:

Uso adecuado de los protocolos de laboratorio/ Apropiación de explicaciones científicas y teorías/ Manejo de procesos de producción/ Identificación de factores que inciden en el trabajo de laboratorio/ Generación de ideas de negocio/ Aplicaciones prácticas de lo aprendido en diferentes contextos/Visión amplia de las posibilidades de trabajo en el campo de la Biotecnología/ Autonomía y emprendimiento: Con iniciativas emprendedoras en la generación de ideas de negocio y posibilidades de desarrollo económico y social/ Conciencia ambiental: Por medio de la línea de producción de abonos orgánicos como resultado del adecuado manejo de los residuos orgánicos con posibilidades agrícolas y económicas.

Se debe señalar además que el trabajo desarrollado desde el campo de la Biotecnología ha transformado las didácticas y metodologías tradicionales que se venían trabajando en el aula, con el surgimiento de nuevas apuestas de aproximación al conocimiento científico.

El colegio ha asumido la Biotecnología como elemento de motivación y proyección laboral, buscando mejorar las prácticas pedagógicas para orientar todos los procesos de aprendizaje y de formación en valores que permitan transformar los enfoques, programas, contenidos y prácticas. En este sentido, cabe mencionar que actualmente el énfasis en Biotecnología forma parte activa de proyectos transversales como el proyecto Ambiental Escolar (PRAE), el proyecto de Lectoescritura, (PLEO) y el Proyecto de Democracia, entre otros, en los cuales se incorporan temas y discusiones relacionadas con la Biotecnología. Adicionalmente, se han articulado curricularmente las Áreas de Ciencias Sociales, Humanidades e Informática para dar apoyo con contenidos específicos requeridos por los estudiantes.

\section{CONCLUSIONES}

1. El énfasis en Biotecnología de la educación media de la jornada nocturna del colegio La Amistad IED se ha constituido en un escenario de inclusión en el que todos los estudiantes han tenido la oportunidad de desarrollar actitudes emprendedoras a partir 
Bio - grafía. Escritos sobre la Biología y su Enseñanza. ISSN 2027-1034

Edición Extraordinaria. p.p. 351 - 362

Memorias del IX Encuentro Nacional de Experiencias en Enseñanza de la Biología y la Educación Ambiental. IV Congreso Nacional de Investigación en Enseñanza de la Biología.

de la propuesta y puesta en marcha de proyectos escolares de emprendimiento relacionados con la Biotecnología y de otras estrategias didácticas.

2. El desarrollo de las estrategias y actividades propias del énfasis en Biotecnología ha permitido establecer que el desarrollo cognitivo va articulado a las oportunidades de relación entre el conocimiento científico y las necesidades sociales de los estudiantes desde un enfoque basado en el aprendizaje situado.

3. La población estudiantil joven contiene una rica experiencia de vida y de saberes populares que deben ser reconocidos desde los marcos de educación formal y específicamente, incorporados en el proceso de enseñanza aprendizaje de las Ciencias Naturales, dado que posibilitan la construcción de conceptos científicos a partir del anclaje con el contexto social y cultural de los estudiantes y posibilitan nuevas oportunidades de vida y de crecimiento humano.

\section{REFERENCIAS BIBLIOGRÁFICAS}

Colegio Distrital La Amistad. (2005). Proyecto Educativo Institucional. Bogotá.

United Nations. (1992). Convention on Biological Diversity, Article 2. Use of Terms.

Frid, D. (2009). Actualidad en Ciencia, Salud, Biotecnología, Medioambiente. Recuperado de: http:// www.tecnocienciaysalud.com

Espinel, N. (2015). Enseñanza de la Biología en América Latina. Revisión de antecedentes. Bio - grafia. Escritos sobre la Biología y su Enseñanza. Número extraordinario, p.1323

Kemmis, S. y MacTaggart, R. (1998) Cómo planificar la investigación acción. Barcelona: Laertes.

Gordillo, M. ( 2009 ). Documento de trabajo No. 03: Educación, Ciencia, Tecnología y Sociedad OEl.

Ministerio de Educación Nacional. Decreto 3011 de 1997.

Morales, J. (2009). Énfasis en Biotecnología, propuesta para la Educación Media.

Orjuela, G. (2013). Integración e Inclusión: Cuestiones de giro y de mirada. UPN 
Bio - grafía. Escritos sobre la Biología y su Enseñanza. ISSN 2027-1034

Edición Extraordinaria. p.p. 351 - 362

Memorias del IX Encuentro Nacional de Experiencias en Enseñanza de la Biología y la Educación Ambiental. IV Congreso Nacional de Investigación en Enseñanza de la Biología.

Padilla, Y.; Gómez-Niño, A.; Villamaría, R., y Sáenz, M. (1997). Proyecto para introducir la Biotecnología en el currículo de la educación secundaria. Enseñanza de las Ciencias, número extra, p. 485-486. Quinto Congreso Internacional de Enseñanza de las Ciencias.

Paz, H. (2007). El aprendizaje situado como una alternativa en la formación de competencias en ingeniería. Revista Educación en Ingeniería. № 4 Diciembre de 2007 pp.1-13. ACOFI.

Posada, J. (2009). Teorías pedagógicas, modelos pedagógicos en la educación de personas jóvenes y adultas y comunidades intelectuales de educadores. UPN.

Secretaria de Educación del Distrito. (2013). Educación Incluyente. Recuperado de: http://www.educacionbogota.edu.co/temas-estrategicos/educacion-incluyente

UNESCO (2005). Guidelines for inclusion: Ensuring Access to Education for All. París: UNESCO Recuperado: http://unesco.org/educacion/inclusive 